\title{
Estudio por imágenes de vía aérea. Utilidad de la tomografía computada multidetector con reconstrucción tridimensional
}

\author{
Airway imaging study. Usefulness of multidetector computed tomography with \\ three-dimensional reconstruction
}

Dr. Alejandra Zaninovic, Dr. José D. Arce, Dra.Sandra Ferrón

Radiólogos. Servicio de Diagnóstico por Imágenes, Clínica Santa María, Santiago, Chile

\begin{abstract}
AIRWAY IMAGING STUDY. USEFULNESS OF MULTIDETECTOR COMPUTED TOMOGRAPHY WITH THREE-DIMENSIONAL RECONSTRUCTION

Introduction. Information obtained by multidetector computed tomography and using reconstruction software now allows precision of intrinsic and extrinsic airway pathological processes. It also shows the pulmonary involvement beyond an obstructed zone. Visualization of different densities with three-dimensional reconstructions facilitates this task. Objective: To show the usefulness of three-dimensional (3D) reconstruction and volume segmentation in airway pathology particularly extrinsic vascular compression. Material and Methods: Pediatric clinical cases studied with Multidetector Computed Tomography are illustrated (suspected airway compression by vascular abnormalities and other causes), in which 3D reconstruction proved to be an important non-invasive diagnostic tool. Studies were performed with Siemens Somatom Definition Team Flash, with 128 channels, with a dual source phase and angiographic technique using low doses of radiation. The reconstructions were performed on a workstation Carestream Vue Pacs. Conclusion: The study of airway with 3D reconstruction and angiographic volume segments allows a better definition of the relation between the compromised airway vessels and other structures on any level in children.
\end{abstract}

Key words: computed tomography, imaging three dimensional, airway, cardiovascular abnormalities

\section{RESUMEN}

Introducción: La información obtenida mediante tomografía computada multidetector (TCMD) y el uso de software para reconstrucción permiten en la actualidad demostrar con exacta precisión los procesos patológicos que comprometen la vía aérea y estructuras extrínsecas que pueden producir compresión. Permite además demostrar el compromiso pulmonar mas allá de una zona obstruida. La posibilidad de visualizar diferentes densidades conjuntamente en reconstrucciones 3D facilita esta labor. Objetivo: Mostrar la utilidad de la reconstrucción tridimensional (3D) en patología de la vía aérea particularmente compresión vascular extrínseca. Material y método: Se ilustran casos clínicos pediátricos examinados con TCMD por sospecha de compresión de la vía aérea por anomalías vasculares y otras causas, en los cuales la reconstrucción 3D demostró ser una importante herramienta diagnóstica no invasiva. Los estudios fueron efectuados en equipo Siemens Somatom Definition Flash, de 128 canales, con una fuente dual en fase angiográfica y utilizando técnicas de baja dosis de radiación. Las reconstrucciones fueron realizadas en una estación de trabajo Carestream Vue Pacs.

Conclusión: En niños el estudio e la vía aérea con reconstrucción 3D y de segmentos de un volumen de un estudio angiográfico con TCMD permite una mejor demostración en cualquier plano de la relación entre la vía aérea comprometida, los vasos y otras estructuras.

Palabras clave: tomografía computada, imagen tridimensional, vía aérea, anomalías cardiovasculares

\section{INTRODUCCIÓN}

La Tomografia Computada Multidetector con reconstrucciones multiplanares y volumétricas (3D), ha demostrado ser una herramienta no invasiva útil para el estudio de la vía aérea, particularmente compresiones extrínsecas de origen vascular. También es útil para la planificación quirúrgica de obstrucciones tumorales intrínsecas o extrínsecas, y estenosis o procesos asociados a malformaciones congénitas $(1,2)$.

\section{Correspondencia:}

Dra Alejandra Zaninovic

Servicio Radiología - Clínica Santa María

Av Santa María 0500 - Torre A

E-mail: jarce@clinicasantamaria.cl
En casos de deformidad de la pared torácica que determinan una redistribución de las estructuras vasculares y vía aérea, puede demostrar tanto la deformidad como sus consecuencias (3-5).

La adquisición de datos en el plano axial puede usarse para interpretar o crear imágenes multiplanares (sagital, coronal, oblicuo) e imágenes 3D y secciones de un volumen determinado, con la posibilidad de visualizar el lumen en forma de una endoscopia virtual. La reconstrucción de imágenes se refiere al proceso de generar imágenes a partir de cortes axiales obtenidos de un espesor muy fino en corto periodo de tiempo, lo que es esencial para el reformateo multiplanar e imágenes 3D. La diferencia de densidad entre las estructuras vasculares y de la vía aérea, generalmente hace que las reconstrucciones 
3D sean efectuadas en forma separada (Figura 1 B, C,E). El desarrollo de los protocolos de reconstrucción que permiten el análisis de diferentes densidades conjuntamente facilitan la demostración del efecto compresivo (Figura 1 D,F).

La existencia de la herramienta que puede estudiar segmentos de un volumen, creando subconjuntos y analizarlos en diferentes planos, permite analizar en forma aislada solo el área de interés, caracterizando en mejor forma la relación entre la vía aérea afectada y la estructura vascular que la comprime, sin superposición de otras estructuras anatómicas (Figura 1 C,F).

Aunque no es nuestro objetivo describir técnicamente el procesamiento de las imágenes disponibles hoy en día con protocolos de reconstrucción, como son MIP (alta densidad), Minimip (baja densidad), SSD (reconstrucción de superficie) 0 volumen rendering (VR), queremos ilustrar a través de casos clínicos, como estas aplicaciones y la reconstrucción 3D permiten una mejor representación de la vía aérea y su relación a las causas de la compresión con énfasis en las anomalías vasculares.

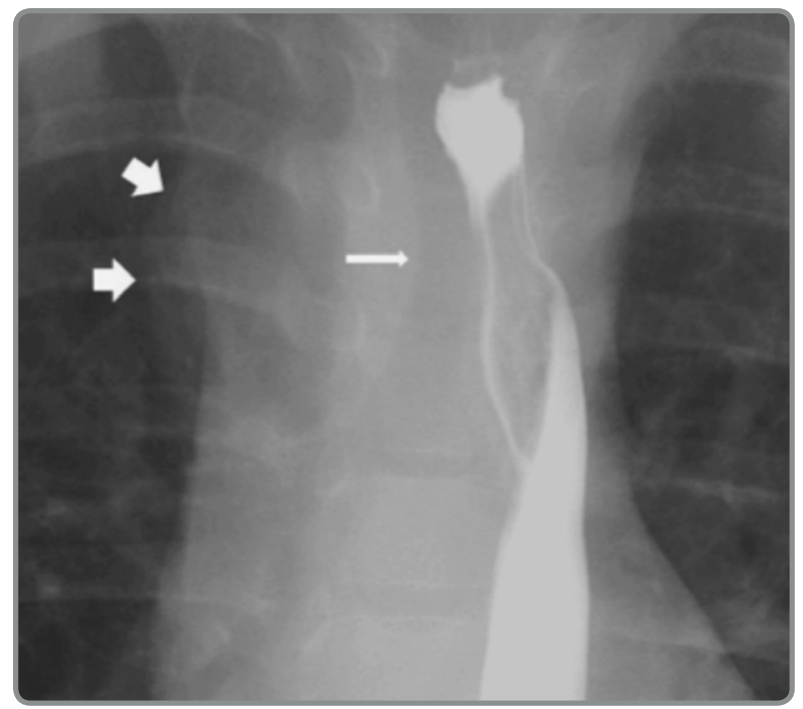

Figura 1. A - F

A. Estudio contrastado del esófago superior demostrando un arco aórtico derecho (flechas gruesas), compresión sobre el borde derecho de la columna aérea de la tráquea (flecha delgada) y defecto por compresión extrínseca del esófago de curso oblicuo y ascendente hacia la izquierda.

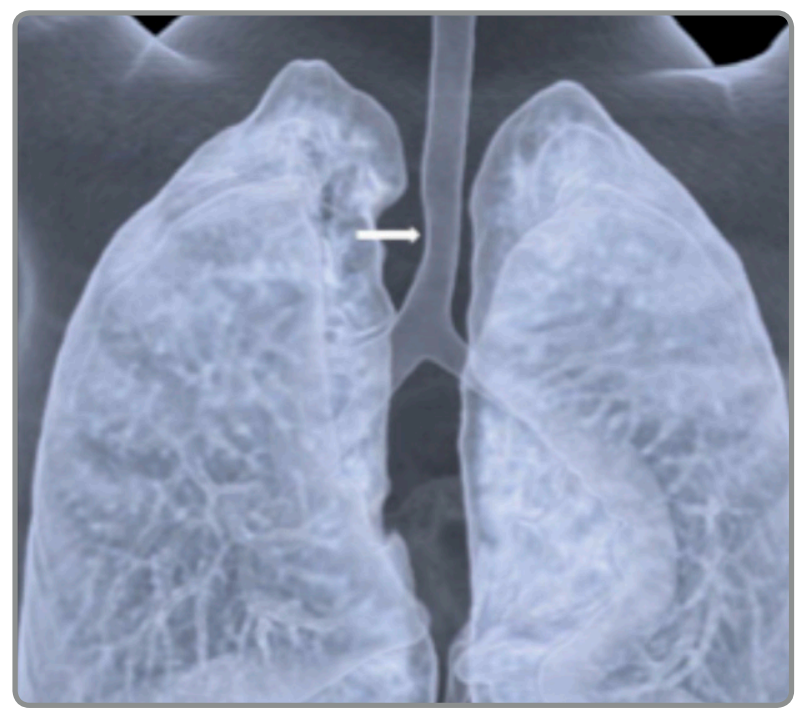

B. Reconstrucción 3D del pulmón y de la vía aérea observándose la compresión de la tráquea (flecha)

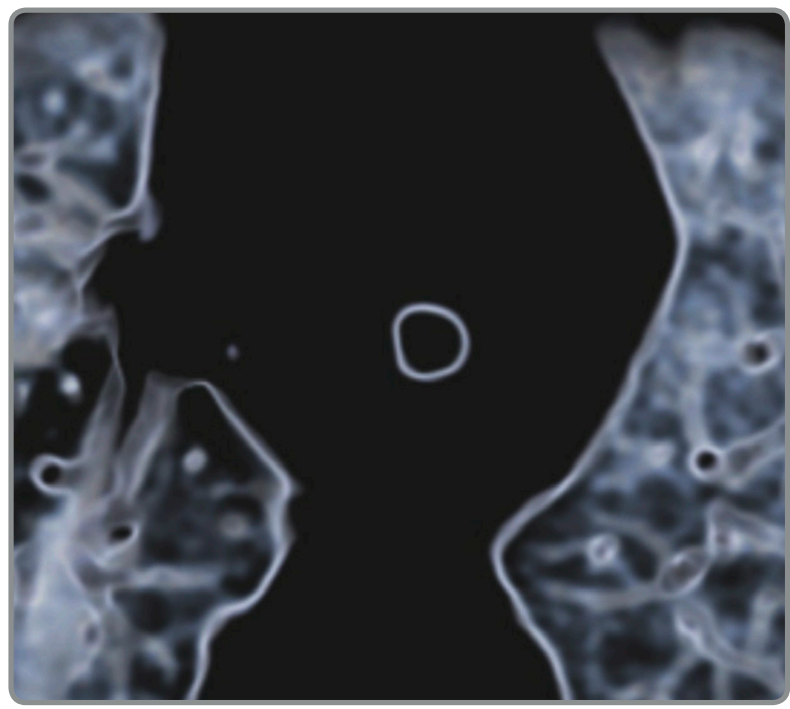

C. Segmento axial oblicuo de la reconstrucción 3D de la vía aérea y del pulmón, a nivel del segmento de la tráquea comprometida. 


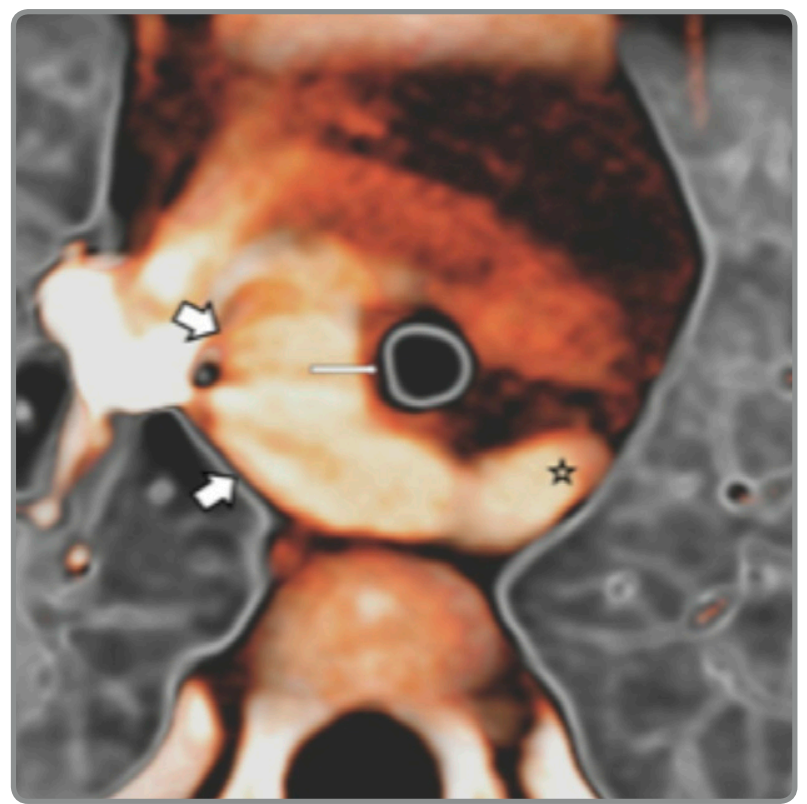

D. Mismo segmento que en $\mathrm{C}$, con superposición de protocolos de reconstrucción de baja y alta densidad, demostrando la impresión del arco aórtico derecho (flechas gruesas) y de la subclavia izquierda aberrante (estrella), sobre la pared lateral y posterior derecha de la tráquea (flecha delgada)

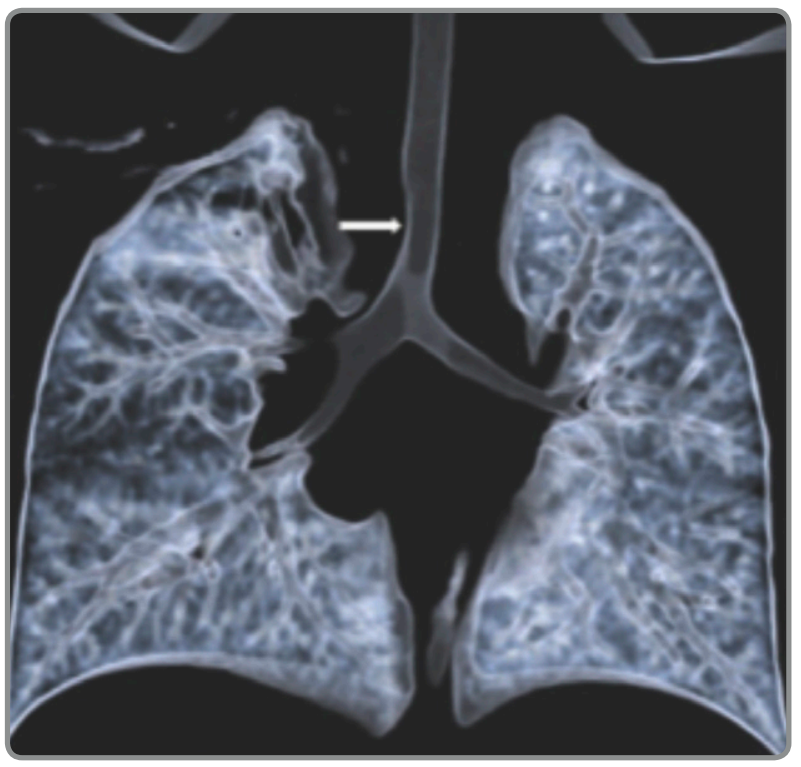

E. Segmento coronal de la reconstrucción de superficie de la vía aérea y del pulmón demostrando en mayor detalle la compresión traqueal.

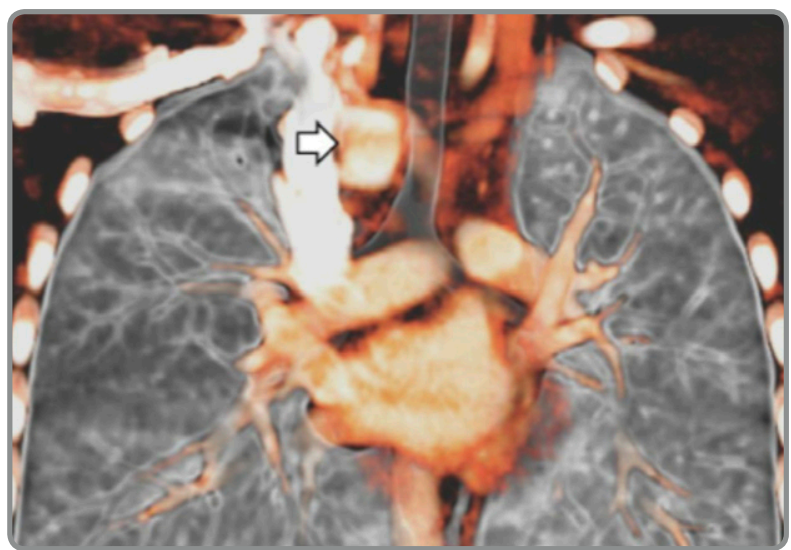

F. El mismo segmento que en E, con superposición de protocolos de baja y alta densidad, demostrando la impresión del arco aórtico derecho (flecha gruesa). Obsérvese por detrás de la tráquea el curso de la subclavia izquierda aberrante.

\section{TÉCNICA DE IMAGEN}

La tomografía computada (TC) de tórax fue realizada en fase angiográfica, en un equipo Somatom Definition Flash, (Siemens Healthcare, Forchheim, Germany) de 128 canales, con una fuente dual, después de la inyección de iodixanol. En nuestros pacientes se usó sedación anestésica, con máscara laríngea

La adquisición fue efectuada con una colimación de 0.6 $\mathrm{mm}$, grosor de corte de $0.75 \mathrm{~mm}$, intervalos de reconstrucción de $0.4 \mathrm{~mm}$, con un pitch de 0,8 , tiempos de rotación de 0.33 y 80 $\mathrm{kV}$.

Los datos fueron transferidos a estaciones de trabajo Carestream Vue Pacs (Carestrem Health Inc, Rochester, USA), permitiendo la reconstrucción y el procesamiento multiplanar y 3D.

\section{CASOS CLÍNICOS}

\section{CASO 1}

Niño de 3 años con sibilancias recurrentes desde el año de edad. Estudios radiológicos previos de tórax, no advirtieron compresión traqueal. El estudio digestivo alto evidenció impresión del esófago anterolateral derecho y posterior por sobre la carina asociado al desplazamiento hacia la izquierda de la tráquea, todos hallazgos sugerentes de un anillo vascular. En la revisión de los antecedentes se informó en una ecografía fetal la presencia de un arco aórtico derecho. Se efectuó el estudio angiográfico con TC multicorte, que demostró el arco aórtico derecho asociado a una arteria subclavia derecha aberrante con compresión traqueal.

El post-proceso de las imágenes tomográficas con reconstruccion $3 \mathrm{D}$ y estudio sectorial, además de la anomalía vascular demostraron la relación con la vía aérea y la deformidad del cartílago traqueal (Figuras 1 y 2) 


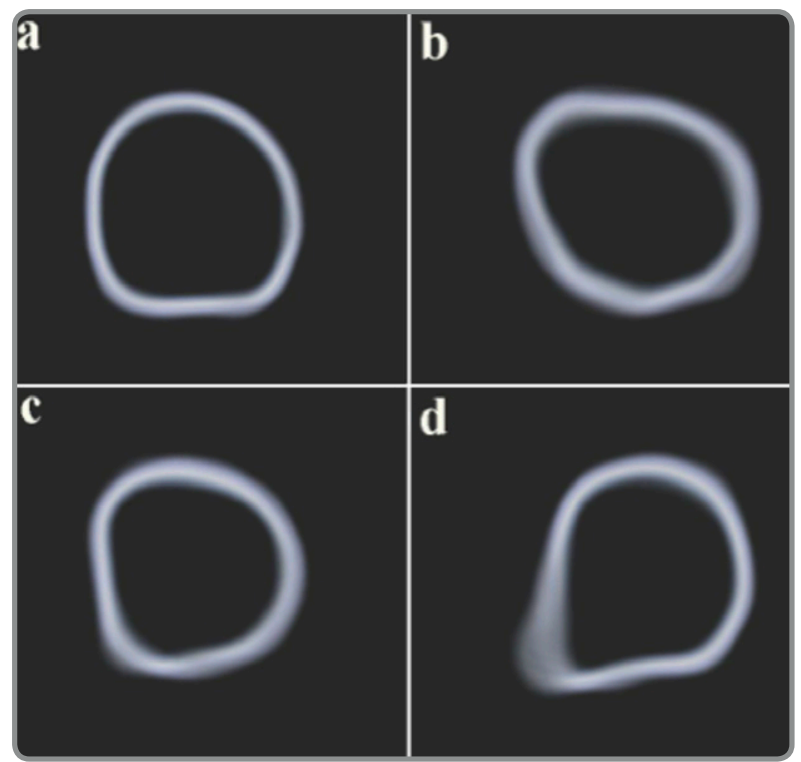

Figura 2.

Magnificación de segmentos de la tráquea, con reconstrucción de superficie de la vía aérea, orientados en planos axiales de la tráquea.

A. Tráquea normal. B - D. Secciones consecutivas del sector de la tráquea comprometida. Obsérvese la deformidad del cartílago lateral y posterior derecho. Cortes en inspiración.

\section{CASO 2}

Recién nacido, género femenino, con síndrome CHARGE, atresia esofágica con fístula la cual es intervenida quirúrgicamente. Evoluciona con fístula broncopleural y dificultad respiratoria. En estudios radiológicos llama la atención la atelectasia del lóbulo superior derecho que alterna con hiperinsuflación.

Se realiza estudio angiográfico con TC multicorte demostrándose un arco aórtico derecho con la arteria subclavia izquierda aberrante, un tronco bicarotideo y un ducto arterioso. El bronquio para el lóbulo superior derecho tiene un curso que está entre la aorta y la rama derecha de la arteria pulmonar. La reconstrucción 3D sectorial demuestra la deformidad bronquial y su relación con las estructuras vasculares comprometidas (Figuras 3 y 4)

Se efectúo cierre de ductus y pexia de la aorta ampliando el espacio del trayecto bronquial. Los controles posteriores del tórax demuestran una adecuada ventilación del lóbulo superior derecho.
Figura 3 A-F. Estudio angiográfico en inspiración.

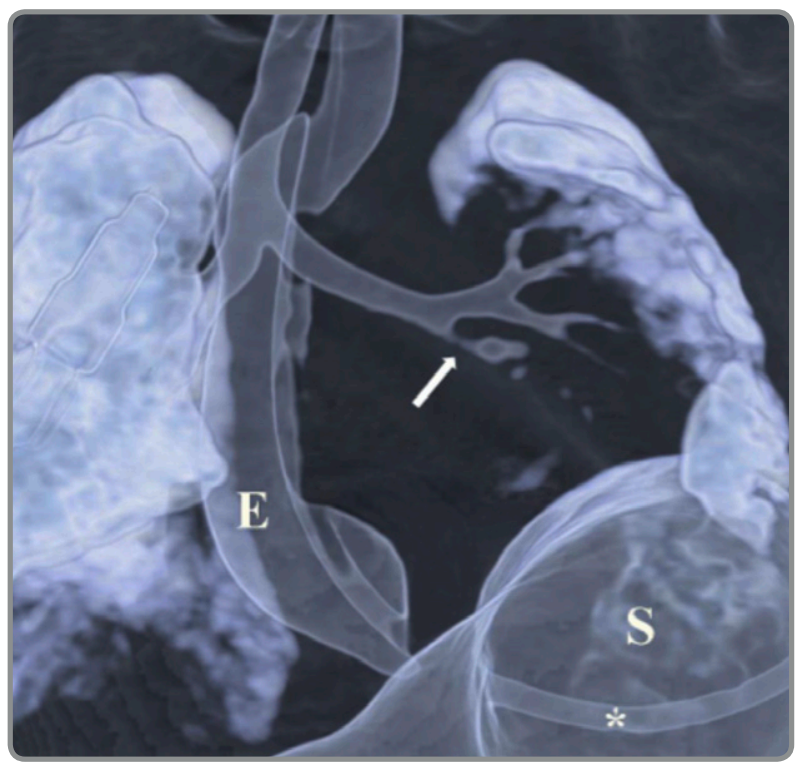

A. Reconstrucción 3D del pulmón y de la vía aérea, observándose la compresión del bronquio para el lóbulo inferior izquierdo (flecha delgada). Nótese además el ascenso del diafragma izquierdo secundario a paresia post-cirugía cardiaca . S: Estómago. E: Esófago. * Sonda nasogástrica.

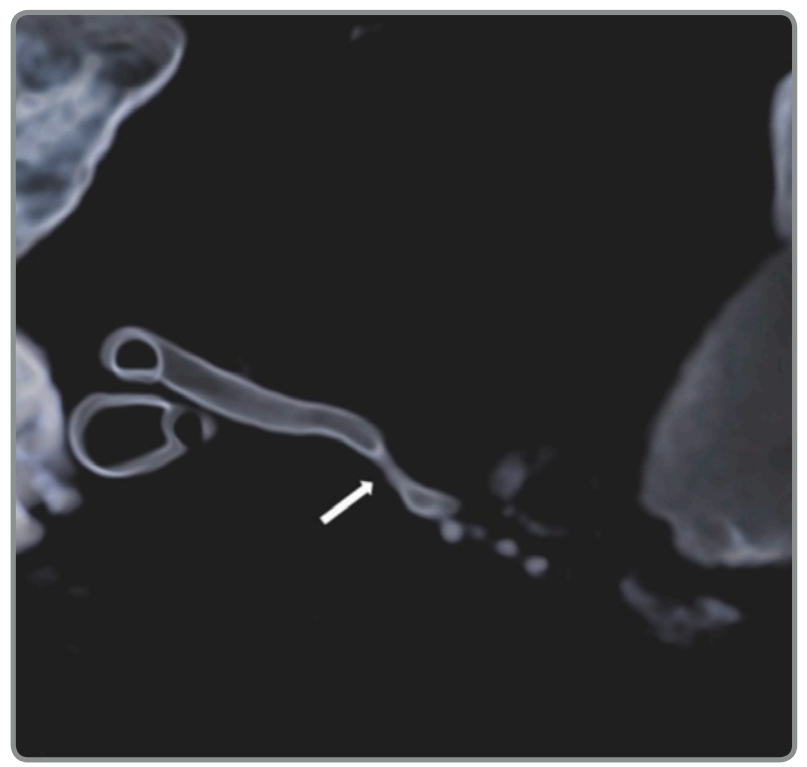

B. Segmento axial oblicuo del tórax, sobre la reconstrucción de superficie de la vía aérea y del pulmón, a nivel del segmento bronquial comprometido. Corte orientado paralelo al trayecto bronquial. 


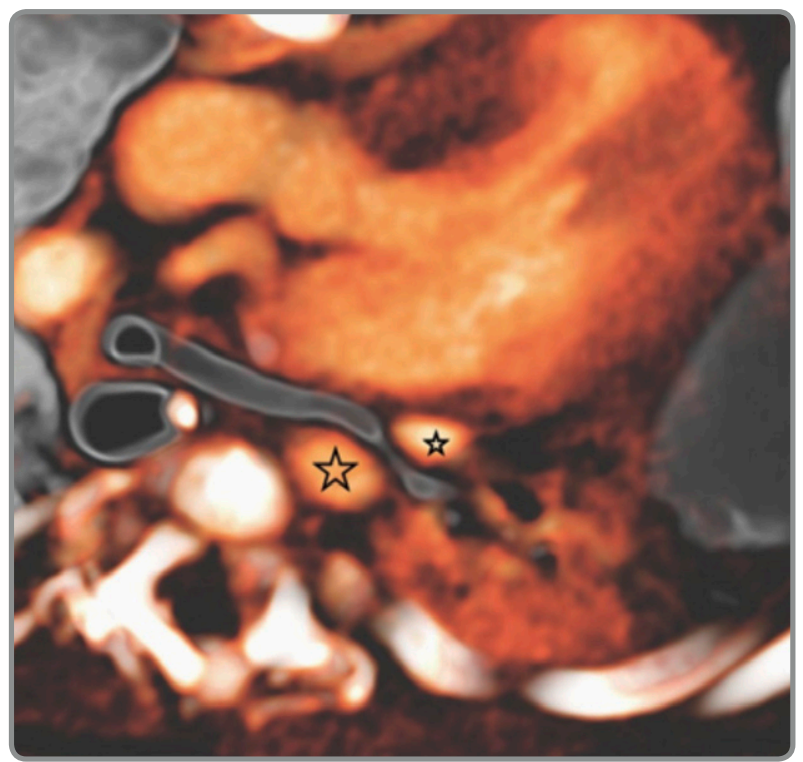

C. El mismo segmento que en $B$, con superposición de protocolos de alta y baja densidad, demostrando la compresión entre la aorta descendente (estrella grande) y la vena pulmonar superior derecha (estrella pequeña)

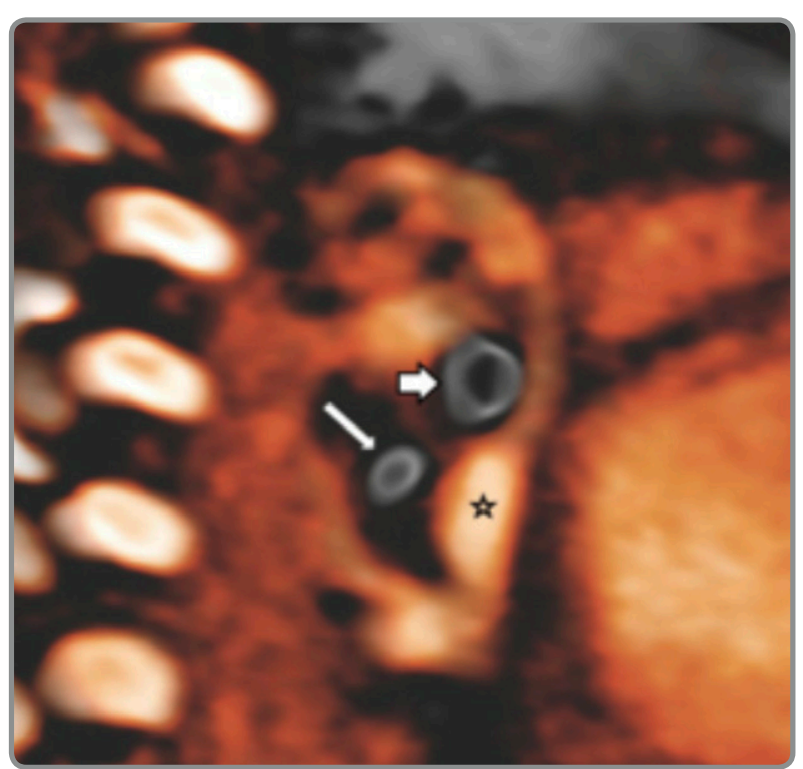

D.

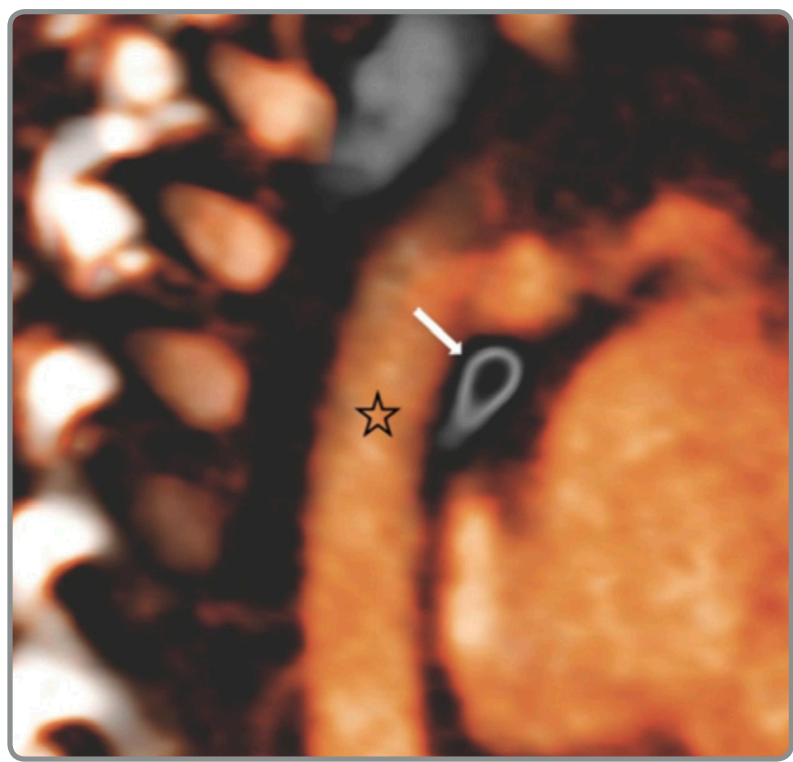

E.

D y E Secciones contiguas, con superposición de protocolos de alta y baja densidad, demostrando la compresión de la aorta descendente (estrella grande) por posterior y de la vena pulmonar superior izquierda por anterior (estrella pequeña).

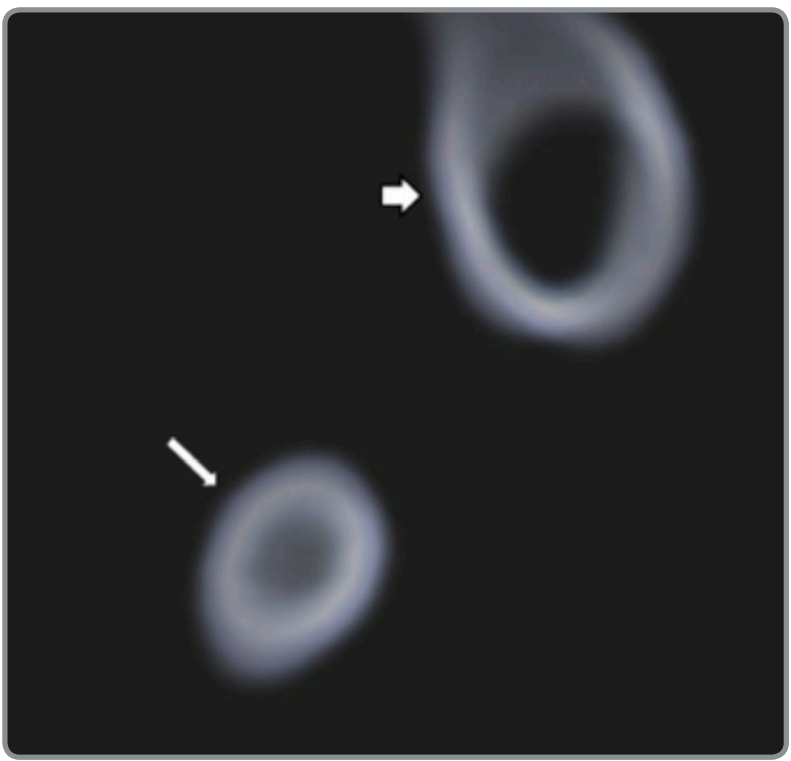

F. Magnificación del segmento del bronquio para el lóbulo inferior izquierdo, orientados en planos estrictamente axial del bronquio (flecha delgada).

Se observa además el bronquio para el lóbulo superior de aspecto normal (flecha gruesa). 
Figura 4. A,B. Estudio efectuado en espiración sin uso de contraste.

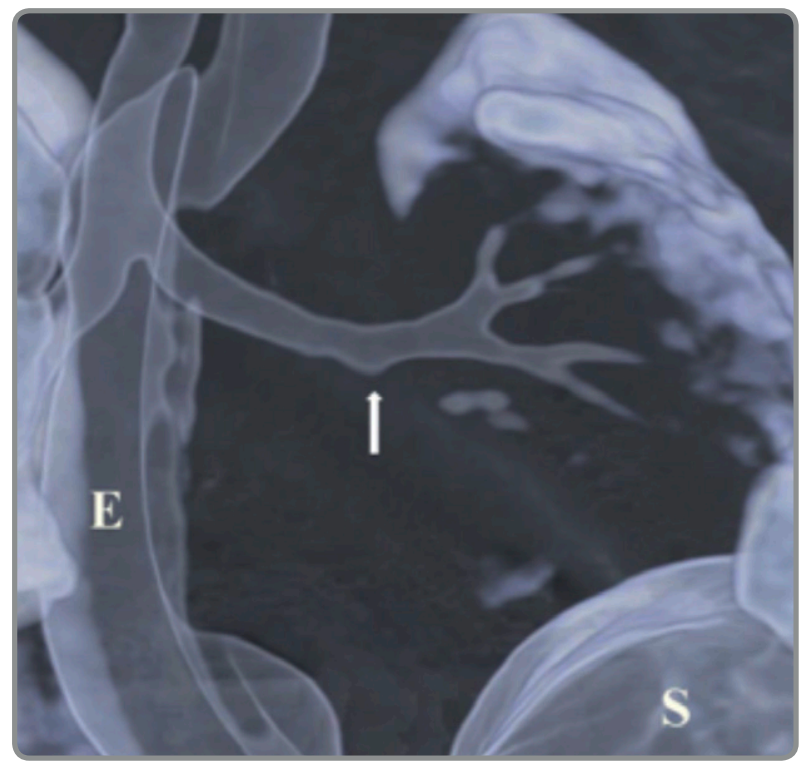

A. Reconstrucción 3D pulmón y vía aérea, observándose la oclusión total del bronquio para el lóbulo inferior izquierdo. (flecha delgada)

(E: Esófago. S: Estomago).

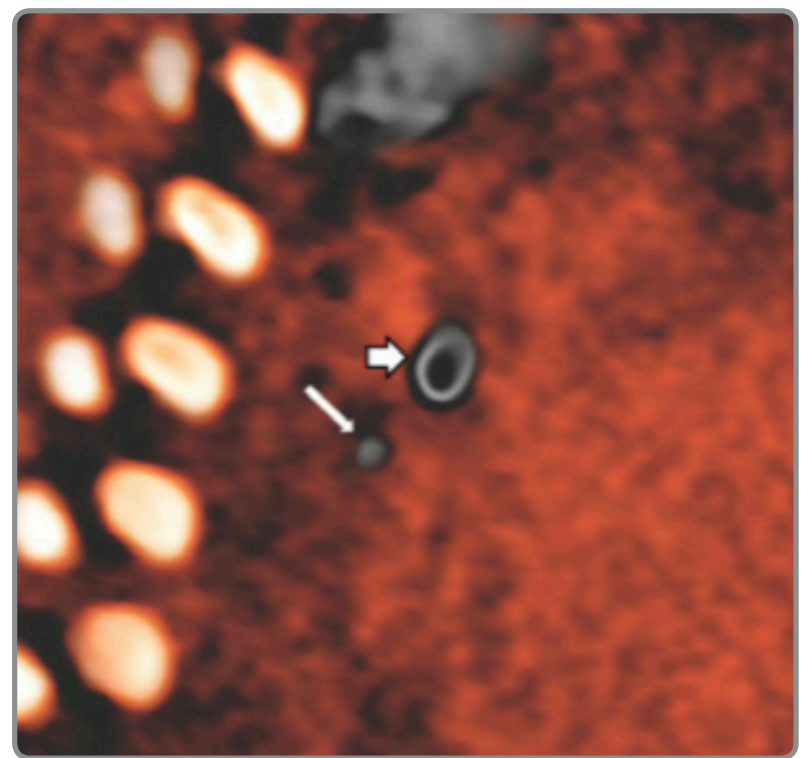

B. Segmento sagital oblicuo del tórax y axial del bronquio para el lóbulo inferior completamente ocluído (flecha delgada). Obsérvese el cambio de calibre del bronquio para el lóbulo superior (flecha gruesa).

\section{CASO 3}

Lactante de 2 meses con cardiopatía congénita: ventrículo único y atresia pulmonar, con un shunt de Blalock. Después de su cirugía se complica con hematoma cerebral frontal, paresia diafragmática izquierda y dificultad respiratoria. El estudio radiológico demostró un ascenso del diafragma izquierdo y atelectasia del lóbulo inferior. El estudio con TC multicorte en inspiración y espiración demuestra además de las alteraciones cardiacas, una obstrucción vascular extrínseca del bronquio para el lóbulo inferior que se objetiva adecuadamente con la reconstrucción 3D sectorial (Figura 5)

Figura 5. A $-\mathrm{F}$

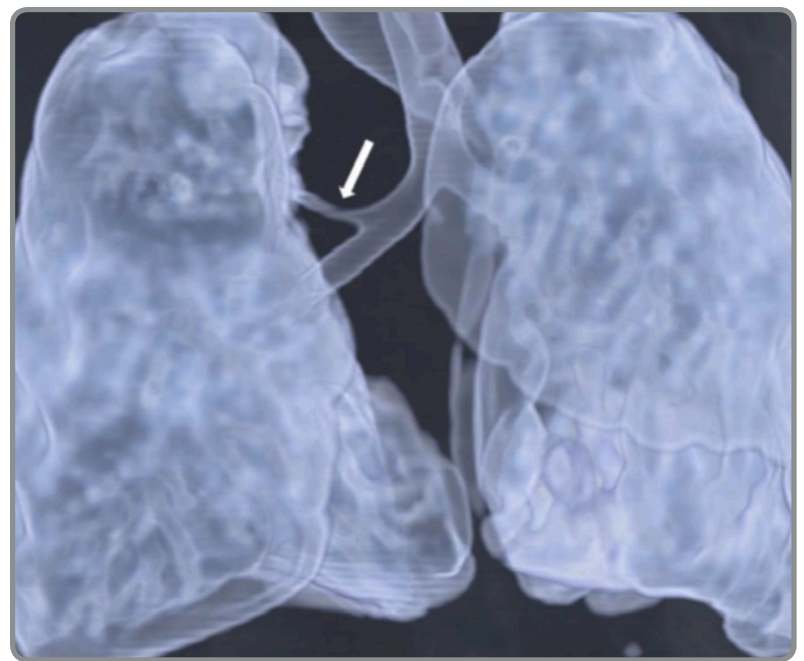

A. Reconstrucción 3D del pulmón y de la vía aérea, observándose la compresión del bronquio para el lóbulo superior derecho (flecha).

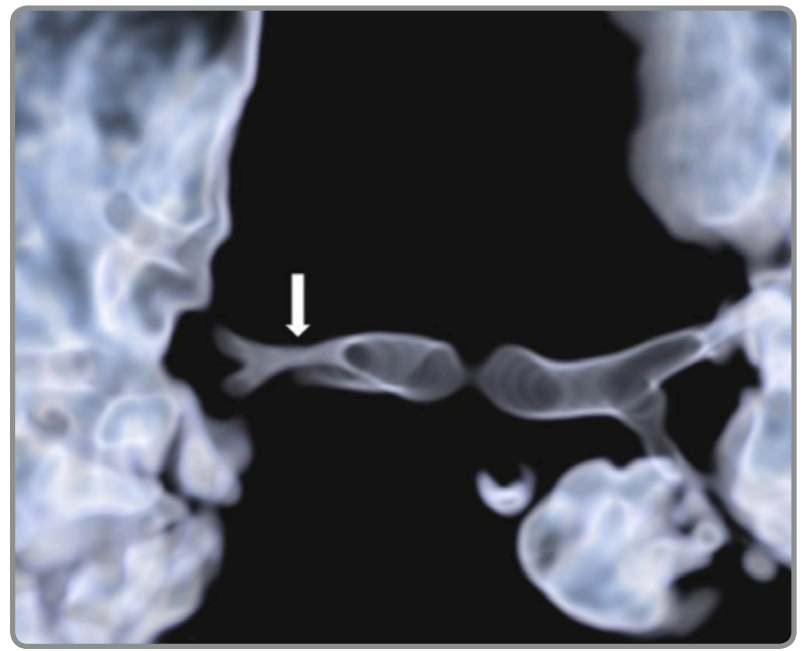

B. Segmento axial oblicuo del tórax, sobre la reconstrucción de superficie de la vía aérea y del pulmón, a nivel del segmento bronquial comprometido (flecha). Corte orientado paralelo al trayecto bronquial.. 


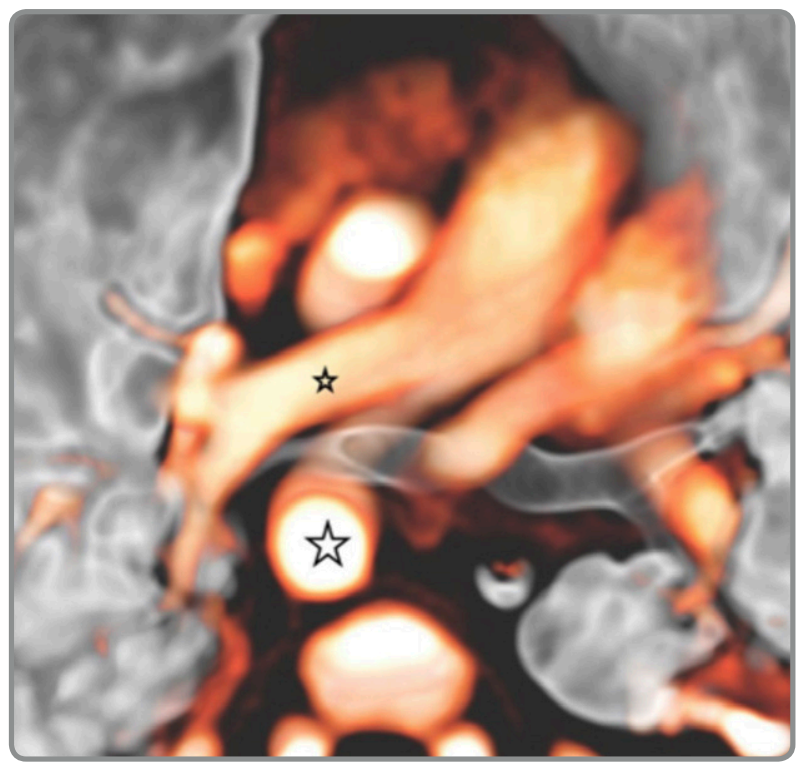

C. El mismo segmento que en $B$, con superposición de protocolos de reconstrucción de superficie y máxima intensidad, demostrando la compresión bronquial entre el arco aórtico derecho (estrella grande) y la rama derecha de la arteria pulmonar (estrella pequeña).

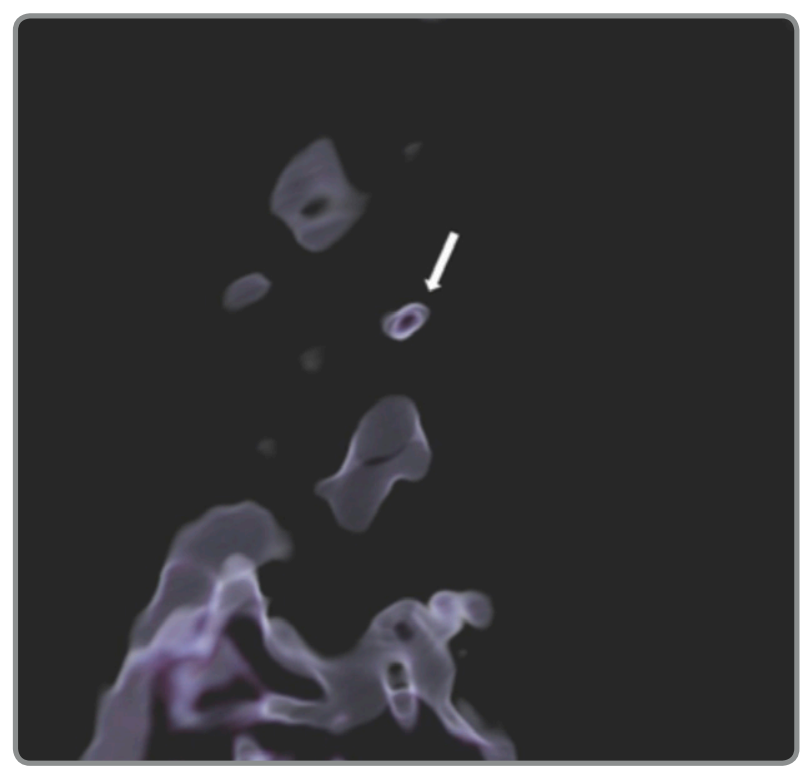

D. Segmento sagital oblicuo del tórax y axial del bronquio para el lóbulo superior, sobre la reconstrucción de superficie de la vía aérea y del pulmón, a nivel del sector bronquial comprometido.

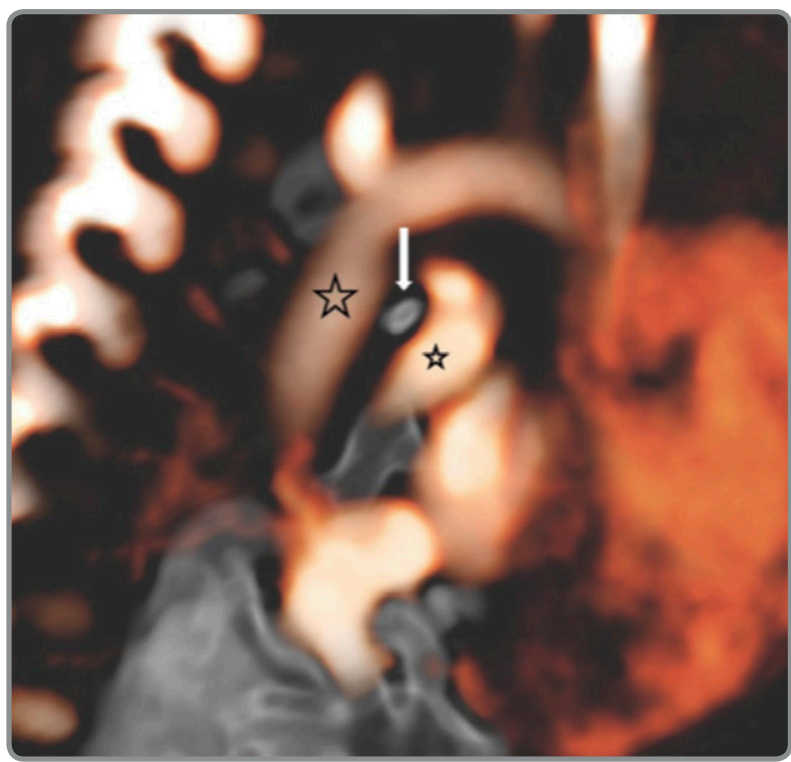

E. El mismo segmento que en $C$, con superposición de protocolos de reconstrucción de superficie y máxima intensidad, demostrando la compresión bronquial entre el arco aórtico derecho (estrella grande) y la rama derecha de la arteria pulmonar (estrella pequeña).

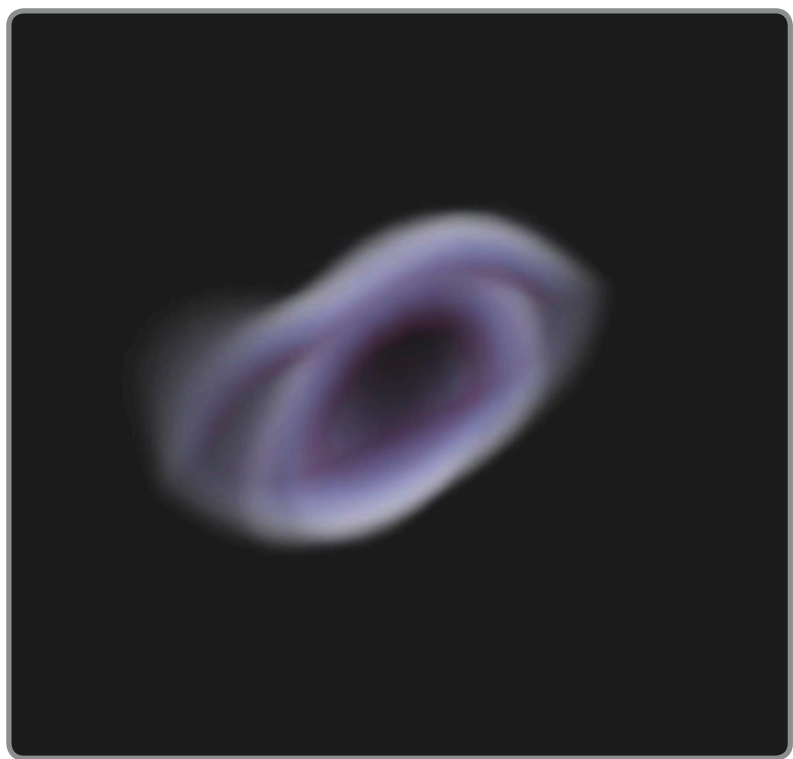

F. Magnificación del segmento del bronquio para el lóbulo superior derecho, de la reconstrucción de superficie de la vía aérea, orientados en planos estrictamente axial del bronquio. 


\section{CASO 4}

Lactante de género masculino de 7 meses con pectus excavatum y cuadros bronquiales obstructivos por lo que se realiza broncoscopia que demuestra estrechamiento del bronquio fuente izquierdo. Se estudia con TC multicorte que evidencia compresión de ambos bronquios principalmente el izquierdo en una larga extensión secundaria al desplazamiento posterior del corazón y las estructuras vasculares por el pectus excavatum (Figura 6).

Figura 6. A-E

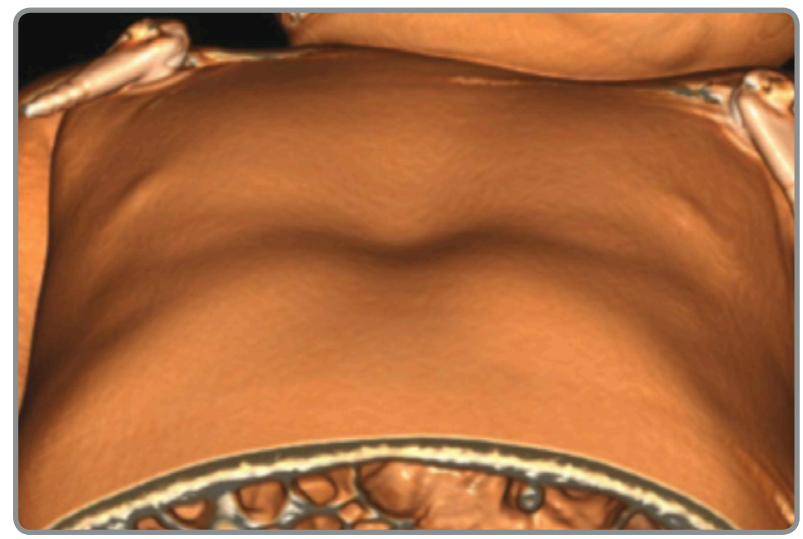

A. Paciente con pectus excavatum. Reconstrucción 3D de la pared toráxica.

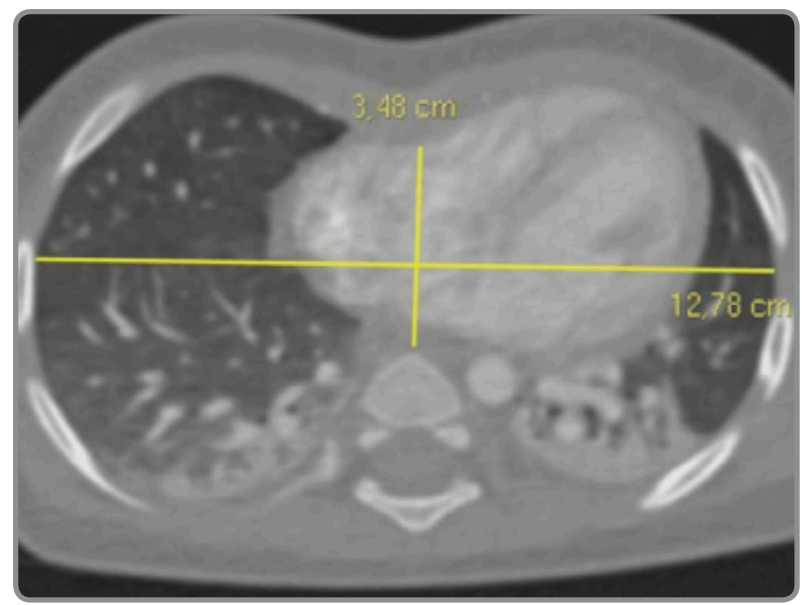

B. Corte axial en ventana pulmonar. Disminución del diámetro anteroposterior del torax y aumento del diámetro transversal. Indice de Haller (Normal: menor de 3,5)

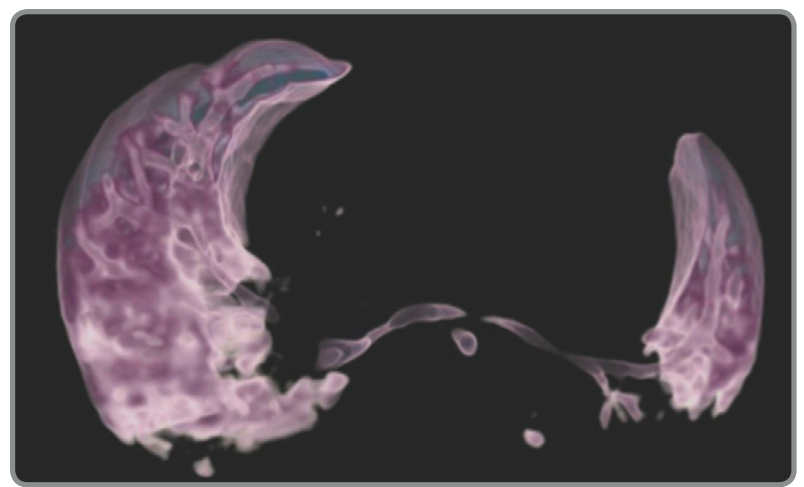

C. Segmento axial de reconstruccion 3D de baja densidad. Desplazamiento posterior de ambos bronquios fuente, mayor a izquierda

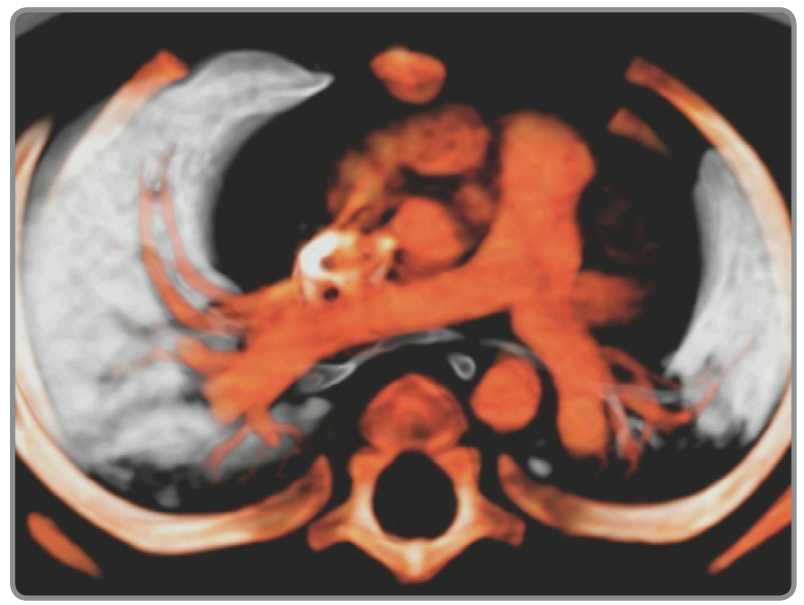

D. Segmento axial de reconstrucción 3D de alta y baja densidad , demostrando la compresión de ambos bronquios secundario al desplazamiento posterior del corazón y de las estructuras vasculares

\section{DISCUSIÓN}

Aún cuando nuestro objetivo fue demostrar la utilidad de la reconstrucción 3D de una tomografía computada multicorte, el estudio inicial de nuestros pacientes es la endoscopía, la cual demuestra malformaciones y estenosis, y además demuestra el compromiso malácico. Sus limitaciones son la demostración de anomalías extraluminales y el compromiso distal a una zona no franqueable (6).

Diversos métodos de imagen pueden emplearse en la evaluación de la anatomía de la vía aérea, y su relación a estructuras vecinas especialmente vasculares. La radiografía de tórax y el esofagograma en algunos casos son una buena aproximación inicial (6). 
La tomografía computada en su estado actual con mínimos tiempos de adquisición de imágenes, facilitan la obtención de información no demostrada por la endoscopía. La imagen tomográfica axial clásica tiene limitaciones tales como la pérdida de evidencia de pequeñas estenosis, la falta de una clara demostración de la extensión craneocaudal de una estructura tubular, la alteración de la anatomía de las estructuras tubulares de disposición oblicua y por último la dificultad para interpretar anatomía compleja. Es por este motivo que la reconstrucción 3D resulta de gran utilidad $(1,3)$.

La MDCT y el uso de reconstrucción 3D nos permiten una detallada demostración de la vía aérea y su relación con las anomalías vasculares del tórax, con la ventaja de ser mínimamente invasiva y de requerir un tiempo extremadamente corto para la adquisición de imágenes. Aunque la desventaja de este método es la dosis de radiación recibida por el niño, es posible reducir el kilovoltaje y miliamperaje utilizado en hasta un $65 \%$ (2) para lograr obtener datos después de inyectado el medio de contraste endovenoso, la que una vez almacenada es información que puede usarse para realizar todos los tipos de reconstrucciones que sean necesarias para un adecuado diagnóstico. Varias publicaciones demuestran su utilidad particularmente relacionados a anillos vasculares y compresión de la tráquea (1-5). Permite visualizar aéreas de estrechamiento focalizado de la vía aérea, la extensión longitudinal de la estenosis, y la vía aérea distal a la zona de estenosis. La Resonancia Magnética también ha sido usada con buenos resultados para evaluar estas anomalías, sin embargo es un estudio que tiene el inconveniente de requerir un prolongado periodo de sedación en niños y la desventaja de una baja resolución espacial $(5,9)$.

En niños cardiópatas, la asociación entre una estructura vascular dilatada, secundaria a un aumento de flujo pulmonar y la compresión de la vía aérea fue propuesta por Rudhe en 1971 (7). Su observación inicial fue para el bronquio del lóbulo medio, sin embargo actualmente se reconoce una particular vulnerabilidad del bronquio para el lóbulo superior izquierdo. Esta anormal relación se facilita en alteraciones del desarrollo del arco aórtico que pueden producir compresión y deformación de la tráquea o en cardiopatías congénitas con aumento del flujo pulmonar, con el mismo efecto en bronquios lobares. Sin embargo en el recién nacido y lactante cualquier alteración en el desarrollo de las estructuras torácicas pueden alterar la normal relación estructura vascular-bronquio como se ilustra en el caso 4, o puede ocurrir por cambios postquirúrgicos, como ocurre en los casos 2 y 3.

El uso de MDTC con cortos tiempos de adquisición, alta resolución espacial, uso de técnicas de baja dosis de radiación y el procesamiento de la información, con reconstrucción 3D ha sido reportada como la mejor técnica de evaluación de la vía aérea asociada a cardiopatías congénitas y anomalías vasculares (1-5). La posibilidad de estudiar secciones de un volumen 3D, facilita la labor particularmente en bronquios lobares, evitando la superposición de estructuras anatómicas, permitiendo examinar el segmento de vía aérea comprometida, en una sección estrictamente axial, independiente de su orientación espacial. El análisis de cortes axiales de la vía aérea, demuestra la exacta alteración anatómica de su lumen, a manera de un estudio endoscópico y permite una mejor evaluación con gran detalle del segmento de vía aérea comprometido. A diferencia del estudio endoscópico, al superponer a la representación axial de la vía aérea, estructuras de alta densidad, se objetiva en forma adecuada los vasos responsables de la compresión, y la presencia de una obstrucción importante tampoco impide la exploración distal a ella. El estudio del segmento comprometido en espiración, manifiesta en mejor forma el colapso de la vía aérea (caso 3).

La aplicación del análisis seccional volumétrico de la vía aérea en cuadros de hiperinsuflación localizada o atelectasia, por compresión extrínseca vascular, permite la evaluación de los bronquios segmentarios ayudando a tomar una adecuada conducta terapéutica. Este método permite también determinar el tamaño del lumen de la vía aérea, grosor de la pared, relación del radio bronquio-arterial, medición de ramas bronquiales distales, demostrando la patología en forma fácil de interpretar para el clínico.

\section{CONCLUSIÓN}

La TC tiene un importante valor diagnóstico en la evaluación de la vía aérea en niños, y especialmente en recién nacidos y lactantes menores en los cuales es fácilmente colapsable por las estructuras vasculares adyacentes, principalmente asociado a cardiopatías congénitas. La posibilidad de obtener buenos estudios angiográficos con TC multidetector utilizando bajas dosis de radiación y el posterior procesamiento de los datos con reconstrucción multiplanar y volumétrica nos permiten su reconocimiento. El postprocesamiento de la información y la utilización de segmentos de un volumen determinado, con o sin visualización interna tipo endoscopía virtual facilitan aún más el estudio detallado de la vía aérea. Estas aplicaciones constituyen una buena alternativa y un eficaz complemento a la utilización de técnicas más invasivas.

Los autores no presentan conflicto de intereses. 


\section{REFERENCIAS}

1. Lee EY, Zucker EJ, Restrepo R, Daltro P, Boiselle PM. Advanced large airway CT imaging in children: Evolution from axial to 4-D assessment. Pediatr Radiol 2013; 43: 285-297

2. Andronikou S, Irving B, Hlabangana LT, Pillay T, Taylor P, Goussard P, Gie R. Technical developments in postprocessing of paediatric airway imaging. Pediatr Radiol 2013; 43: 269-2841

3. Ramos-Duran L, Nance Jr. JW, Schoepf UJ, Henzler T, Apfaltrer P, Anthony M. Hlavacek AM. Developmental Aortic Arch Anomalies in Infants and Children Assessed With CT Angiography. AJR 2012; 198: W466-W474

4. Zhong YM, Jaffe RB, Liu JF, Sun AM, Gao W, Wang Q, Zhu M, Qiu HS, Berdon WE. Multi-slice computed tomography assessment of bronchial compression with absent pulmonary valve. Pediatr Radiol 2014; 44: 803-809

5. Dillman JR, Attili AK, Agarwal PP, Dorfman AL, Hernandez RJ, Strouse PJ. Common and uncommon vascular rings and slings: a multi-modality review. Pediatr Radiol 2011; 41: 1440-1454

6. Kussman BD, Geva T, McGowan FX. Cardiovascular causes of airway compression. Pediatric Anesthesia 2004;14: 60-74

7. Collins LK, Levin TL, Walter E. Berdon WE, Cowles RA, Newman B. Rudhe syndrome: reversible right middle lobe emphysema in infants with left-to-right shunts-an historical review. Pediatr Radiol 2010; 40: 762-765

8. Taragin BH, Berdon WE, Printz B. MRI assessment of bronchial compression in absent pulmonary valve syndrome and review of the syndrome. Pediatr Radiol 2006; 36: 71-75

9. Lee EY, Browne LP, Lam W. Noninvasive magnetic resonance imaging of thoracic large vessels in children. Semin Roentgenol 2012; 47:45-55 